\title{
Core-Shell Structured Si/ZnO Photovoltaics
}

\author{
Mengyao Zhang ${ }^{\# 1} *$, Xianfeng Gao ${ }^{\# 2}$, Anthony Barra ${ }^{2}$, Paichun Chang ${ }^{3}$, Liubing Huang ${ }^{1}$, \\ Robert Hellwarth ${ }^{2}$, Jia Grace $\mathrm{Lu}^{2}$ \\ ${ }^{1}$ Mork Family Department of Chemical Engineering and Materials Science, ${ }^{2}$ Department of Physics \& \\ Astronomy, and Ming Hsieh Department of Electrical Engineering, University of Southern California, Los \\ Angeles, CA 90089-0484, USA. \\ ${ }^{3}$ School of Informatics, Kainan University, Taoyuan 33857, Taiwan \\ *E-mail: mengyaoz@usc.edu \\ \# These authors have contributed equally
}

\begin{abstract}
Periodic Si pillar arrays synthesized by metal assisted chemical etching method exhibit an excellent light harvesting capability, ideal for core-shell structured solar cell applications. To investigate the photovoltaic prospective, a radial heterojunction device is fabricated by conformal coating a layer of $\mathrm{Al}$ doped $\mathrm{ZnO}$ film on the $p$-Si pillar array. This core-shell structure has achieved low reflectance $(<12 \%)$ over a broad wavelength range, and has demonstrated more than doubled enhancement of the short-circuit current as compared to the traditional planar architecture. Although reverse recovery transient measurement shows that this device with no surface passivation has shorter minority carrier life time than the planar structure, the overall efficiency is enhanced. It suggests that device efficiency could be much improved through proper surface treatment. This renders a promising path for core-shell structured solar cell with higher efficiency yet reduced material per device element.
\end{abstract}

Keywords: Silicon solar cell; core shell structure; resonant absorption; carrier lifetime

\section{Introduction}

Solar energy is one of the abundant, clean and sustainable energy sources. In recent years, much research effort has been directed to develop cost-effective photovoltaic devices through configuration and material innovations. To build a high efficient solar cell, all three essential processes in photoelectric energy conversion should be concurrently optimized, i.e. light absorption, charge separation and charge collection. However, light 
absorption and charge separation are difficult to be fulfilled simultaneously in traditional planar structured solar cells. Thick absorption layer results in better light harvest but less efficient charge collection, limited by the minority diffusion length in the material. Coreshell photovoltaic structure provides a viable solution to this issue and has attracted considerable interest because it orthogonalizes the light absorption and charge collection direction. In addition, it possesses many advantages including reduced reflection, enhanced light trapping capability, increased defect tolerance, reduced material cost, and large area scalability. ${ }^{[1-6]}$

Inspired by the three dimensional pillar structures, we have constructed a prototype of $p$ $\mathrm{Si} / n-\mathrm{ZnO}$ heterojuntion solar cell. $\mathrm{Si}$ is extensively utilized because of its abundance, stability, low toxicity, and high efficiency-to-cost ratio. Yet, as one knows, most of the energy $(>70 \%)$ in the UV region is lost in Si through lattice thermalization due to its narrow energy bandgap $(1.12 \mathrm{eV})$, resulting in increased operation temperature and degrading device performance. In order to promote more efficient UV light absorption, $\mathrm{ZnO}$ with a direct wide bandgap $(3.37 \mathrm{eV})$ is selected as the complementary material for its high absorption in the UV region. ${ }^{[7]}$ In addition, $\mathrm{ZnO}$ has high transmittance in the visible region and relatively high electric conductivity, and is often utilized as the antireflective window layer in solar cells. ${ }^{[7-9]}$ Moreover, $\mathrm{Si} / \mathrm{ZnO}$ heterojunction solar cell is theoretically predicted to have a conversion efficiency as high as $25 \% .^{[10]}$ In this work, periodic Si pillar array is used to form $p-\mathrm{Si} / n-\mathrm{ZnO}$ photovoltaic, not only because it reduces the active material volume, but more importantly, it also maximizes the optical absorption in contrast to $\mathrm{Si}$ thin film planar configuration. ${ }^{[1,12]}$ This study aims to evaluate the core-shell structured geometry on the performance of the radial heterojunction photovoltaic device. The electrical, optical and photovoltaic properties of the prototype are investigated. Comparison is made to its planar structured counterpart in order to address the fundamental property differences between perpendicular and periodic radial junctions.

\section{Experiments}


Periodic $p$-Si pillar arrays were fabricated by a simple metal-assisted chemical etching method, ${ }^{[13]}$ and the periodicity was controlled by pre-patterning of metal catalyst through optical lithography. $p$-Si wafer (boron-doped (110) thickness $\sim 400 \mu \mathrm{m}$ ) substrate was first cleaned by acetone, ethanol and DI water, and then quickly dipped in HF solution for 30 seconds to remove the native oxide on the surface. Photoresist coating with periodic dot structure was patterned via photolithography on the Si substrate, followed by a bilayer catalyst $(\mathrm{Ag} / \mathrm{Au}, 20 \mathrm{~nm} / 5 \mathrm{~nm})$ deposition. After removing the unwanted part of the metal layer, (those on the top of dot structured photoresist) Si wafers were etched in the etchant solution $\left(0.02 \mathrm{M} \mathrm{H}_{2} \mathrm{O}_{2}, 5 \mathrm{M} \mathrm{HF}\right)$ at room temperature. During the etching, $\mathrm{Ag}$ served as a local cathode to promote the reduction of oxidant $\left(\mathrm{H}_{2} \mathrm{O}_{2}\right)$ and the generation of holes at the metal-semiconductor interface, followed by the reaction with anode ( $\mathrm{Si}){ }^{[14]}$ The top Au layer was to protect the Ag film from dissolving in etchant. ${ }^{[13,15]}$ Thus, the region with metal coating is etched down faster than dot region and a pillar structure is formed. Then the samples were cleaned by DI water, and the residual metal was removed by $\mathrm{H}_{2} \mathrm{SO}_{4}$ and $\mathrm{H}_{2} \mathrm{O}_{2}$ mixture.

To form core-shell structures, the as-synthesized Si pillar arrays were coated with $\mathrm{ZnO}$ : Al film (highly $n$-doped) by RF magnetron sputtering. Meanwhile, a bare Si wafer was coated in the same process batch to fabricate planar structured solar cells for comparison study. Before $\mathrm{ZnO}$ deposition, $\mathrm{Ar}^{+}$plasma was applied to the samples to remove the native oxide and to reduce the contact serial resistance. The RF power was set at $150 \mathrm{~W}$ during the sputtering process while the deposition time was 1.5 hours. To improve the crystal quality of the $\mathrm{ZnO}$ layer, a post annealing process was carried out in Ar ambience at $500{ }^{\circ} \mathrm{C}$ for 1 hour.

The morphology of the samples was characterized by a field-emission scanning electron microscope (FESEM, JEOL JSM-7001F). Reflection spectra were obtained by a UV/Vis/NIR Spectrophotometer (LAMBDA 950) with an integrating sphere. The photocurrent density versus voltage $(\mathrm{J}-\mathrm{V})$ characteristics measurements were performed by a semiconductor parameter analyzer (HP 4156c) under simulated sunlight (AM 1.5G, $100 \mathrm{~mW} / \mathrm{cm}^{2}$ produced by an Oriel Solar Simulator). The metal contacts were selected to 
be $\mathrm{Ni} / \mathrm{Au}$ for $p$-Si and $\mathrm{Ti} / \mathrm{Au}$ for $n-\mathrm{ZnO}$ in order to minimize the work function mismatch. Minority carrier life time of the $p-\mathrm{Si} / n-\mathrm{ZnO}$ heterojunction was investigated by reverse recovery transient method, where voltage was applied by a digital delay/pulse generator (DG535) and current response was monitored by an oscilloscope (Agilent MSO8104A).

\section{Result and Discussion}

\subsection{Morphology of Si micro pillar arrays and Si/ZnO heterojunction}

Figure 1 shows the SEM images of well-ordered Si pillar arrays. The diameter of each pillar is $\sim 2 \mu \mathrm{m}$ with a pitch size of $\sim 5 \mu \mathrm{m}$ between adjacent pillars. The dimensions can be adjusted for optimization of light scattering and absorption. And the height of pillars can be controlled by adjusting the etching time. As shown in Fig. 1b-d, the height of Si pillars is $0.68 \mu \mathrm{m}, 1.15 \mu \mathrm{m}$, and $2.8 \mu \mathrm{m}$, corresponding respectively to different etching time of $7 \mathrm{~min}, 15 \mathrm{~min}$ and $30 \mathrm{~min}$. In this prototype evaluation, Si pillar arrays with a height of $0.68 \mu \mathrm{m}$ have been selected to construct $p-\mathrm{Si} / n-\mathrm{ZnO}$ core-shell structures. The conceptual comparison between the radial junction core-shell structure and the planar structure is illustrated in Fig 2a. Figure 2b shows the cross-section SEM images of coreshell structure in contrast with the planar structure $p-\mathrm{Si} / n-\mathrm{ZnO}$ heterojunctions (Fig. 2c), illustrating a large area conformal coating of $\mathrm{ZnO}$ film (thickness $\sim 600 \mathrm{~nm}$ ) on the $\mathrm{Si}$ pillar arrays.

\subsection{Reflectance measurement}

Figure 3 shows the total reflectance spectra of Si wafer and periodic Si pillar array with and without $\mathrm{ZnO}$ film coating at a wavelength range of $300-800 \mathrm{~nm}$. Note that the transmission is negligible for the thickness of Si substrate $(\sim 400 \mu \mathrm{m})$. Compared with the planar Si wafer, Si pillar array shows $\sim 30 \%$ lower reflectance over the whole spectrum. The vertically aligned pillar array fosters the resonant trapping of light in the periodic structure. ${ }^{[16]}$ In addition, it facilitates photons to scatter within the array for higher probability of absorption. ${ }^{[12,16]}$ It is also observed that with $\mathrm{ZnO}$ film coating, both planar Si and Si pillar array show a noticeably decrease in reflection, especially in the UV 
region $(300-400 \mathrm{~nm})$, owing to lower refractive index of $\mathrm{ZnO}\left(n_{Z n O^{\sim 2}}\right)^{[8]}$ compared with $\mathrm{Si}\left(n_{S i} \sim 5\right)$, as well as high UV absorption nature of $\mathrm{ZnO}$. Meanwhile, significant oscillations appear in the reflection of planar $\mathrm{Si} / \mathrm{ZnO}$ device, resulting from thin film interference in the $\mathrm{ZnO}$ coating layer. The reflection peaks $(745 \mathrm{~nm}, 571 \mathrm{~nm}, 471 \mathrm{~nm}$ and $414 \mathrm{~nm})$ match the constructive interference condition $2 d n_{\mathrm{ZnO}}(\lambda)=k \lambda(\lambda$ is the wavelength, $d \sim 600 \mathrm{~nm}$ is the film thickness and $n_{\mathrm{ZnO}}(\lambda)$ is the refractive index of $\mathrm{ZnO}^{[8]}$ ). In contrast, in Si pillar/ZnO device, the interference was significantly suppressed. Taking advantage of both periodic pillar structure and $\mathrm{ZnO}$ film, low reflectance $(<12 \%)$ over the whole wavelength is observed in core shell structured device. (Figure 3 green line) In summary, the core-shell $p-\mathrm{Si} / n-\mathrm{ZnO}$ shows far superior light harvest capability than the planar structure, demonstrating the potential for improving the energy conversion efficiency.

\subsection{Minority carrier life time}

To elucidate the minority carrier lifetime of the core-shell structured devices, reverse recovery transient (RRT) measurement was performed from which the average lifetime of minority carriers in both $p$ and $n$ regions can be extracted by analyzing the transient current response after the voltage bias is abruptly reversed. At forward bias, excess minority carriers are stored in the quasi-neutral region forming a diffusion capacitance. ${ }^{[17]}$ After sudden reversing the bias voltage, since voltage across capacitor cannot change instantaneously, the reverse current $I_{r}$ remains constant for a certain period of time, so called the storage time $\left(t_{s}\right)$. Then the current starts to decay until the excess minority carries are recombined as the current flow out of the quasi-neutral region. Figure 4 shows the RRT measurements on both planar structure and core-shell structure under a range of forward and reverse bias voltages ( -2 to $2 \mathrm{~V},-2$ to $1 \mathrm{~V}$, and -3 to $1 \mathrm{~V}$ ). While changing the forward and reverse bias voltages, the forward and reverse currents vary and induce different diffusion capacitance, yielding different storage time for excess carrier recombination. The relationship between minority carrier lifetime $\tau$, storage time $t_{s}$, forward current $I_{f}$, and reverse current $I_{r}$ is known as ${ }^{[18]}$ 
The storage time $t_{s}$ is estimated by determining the inflection point of the curve. The minority carrier life time $\tau$ can then be extracted from the slope of $t_{s}$ versus $\ln \left(1+I_{f} / I_{r}\right)$ curve, as shown in the insets of Figure 4. The minority carrier lifetimes are calculated to be $197 \mathrm{~ns}$ for planar (Figure 4a) and $135 \mathrm{~ns}$ for core-shell $p$-Si/n-ZnO structure (Figure 4b). A shorter minority carrier diffusion length in the core-shell structure could be originated from the dangling bonds on the surface of Si pillars acting as recombination centers ${ }^{[19]}$ and the increased trapping site density due to enlarged surface area. ${ }^{[20,21]}$ Such hindering effect of increased surface recombination also exists in various nanowire structured devices. ${ }^{[12,18,22,23]}$

\subsection{Photovoltaic performance of $\mathrm{Si} / \mathrm{ZnO}$ devices}

The energy band diagram of $p-\mathrm{Si} / n-\mathrm{ZnO}$ junction is illustrated in Figure 5a, showing photo-generated electron-hole pair separation at the depletion region. The current density $v s$ voltage $\mathrm{J}-\mathrm{V}$ characteristics of the planar and core-shell structure $p-\mathrm{Si} / n-\mathrm{ZnO}$ solar cell are plotted in Figure 5b. All the samples show rectifying behavior, confirming $p-n$ junction formation at the $p-\mathrm{Si} / n-\mathrm{ZnO}$ interface. The inset of Figure $5 \mathrm{~b}$ displays magnified $\mathrm{J}-\mathrm{V}$ curves of both devices under light illumination. The planar structured solar cell shows an open circuit voltage $\left(V_{O C}\right)$ of $16 \mathrm{mV}$, a short circuit current density $\left(J_{S C}\right)$ of 0.72 $\mathrm{mA} / \mathrm{cm}^{2}$ and photoelectric conversion efficiency $(\eta)$ of $0.58 \%$. In comparison, for the core-shell structured solar cell, $J_{S C}=1.92 \mathrm{~mA} / \mathrm{cm}^{2}, V_{O C}=9 \mathrm{mV}$ and $\eta=0.86 \%$. The fill factor for both planar structured and core-shell structured solar cells is $25 \%$. The significantly increased short circuit current density in the core-shell structured solar cell is a result of the remarkable enhancement in light absorption. On the other hand, $V_{O C}$ is decreased in the core-shell structured device. $V_{O C}$ is the voltage at which the diffusion current balances out generated of photocurrent. $V o c=V_{a}(I=0)=\frac{K T}{e} \ln \left(\frac{I_{p h}}{I_{s}}+1\right)$, where $I_{s}$ is the reverser saturation current of $p$-n junction and $I_{s}=q A\left(\frac{e D_{p} p_{n 0}}{L_{p}}+\frac{e D_{n} n_{p 0}}{L_{n}}\right)$ with $L_{p}$ and $L_{n}$ represent the minority carrier mean free path.. ${ }^{[17]}$ Thus, the decrease in $V_{O C}$ can be attributed to the increased number of recombination sites and shorter minority carrier 
lifetime as depicted in the reverse recovery transient measurements. Nevertheless, the overall efficiency was enhanced by $50 \%$ in the core-shell structure, primarily owing to the superior light absorption capability. The efficiency of core-shell structure could be further enhanced by improving interface junction quality, which can be achieved by passivating the surface of the Si pillars. ${ }^{[24]}$

\section{Conclusion}

Core-shell structured $p-\mathrm{Si} / n-\mathrm{ZnO}$ heterojunction photovoltaic devices are fabricated via a straightforward fabrication process based on patterned vertical Si pillar arrays. The periodic array optimizes light absorption, and the radial heterojunction geometry serves to decouple the incident light from the charge separation direction. This device prototype has demonstrated substantial improvement in photocurrent density as compared to the planar structured solar cells. Meanwhile, RRT measurement suggests that the core-shell structure with increased surface-to-volume ratio facilitates higher surface charge recombination rate. The device shows overall enhanced efficiency even without further surface engineering. In summary, this device geometry offers a promising design for developing simple to fabricate, cost reducing and large area scalable solar cells.

\section{Acknowledgement}

This work is supported by the Center for Energy Nanoscience funded by the U. S. Department of Energy, Office of Science, Energy Frontier Research Center (EFRC) program under Award Number DE-SC0001013. The authors thank Chia-chi Chang for assistance of RRT measurement, and Dr. Siyuan Lu for assistance in solar cell J-V measurement. 


\section{References}

[1] Tian B, Zheng X, Kempa TJ, Fang Y, Yu N, Yu G, et al. Coaxial silicon nanowires as solar cells and nanoelectronic power sources. Nature. 2007;449:885-9.

[2] Kayes BM, Atwater HA, Lewis NS. Comparison of the device physics principles of planar and radial p-n junction nanorod solar cells. Journal of Applied Physics. 2005;97:114302.

[3] Garnett EC, Yang P. Silicon Nanowire Radial p-n Junction Solar Cells. J Am Chem Soc. 2008;130:9224-5.

[4] Zhang M, Wang Y-N, Moulin E, Grützmacher D, Chien C-J, Chang P-C, et al. Core-shell CdTe-TiO2

nanostructured solar cell. Journal of Materials Chemistry. 2012;22:10441.

[5] Garnett EC, Brongersma ML, Cui Y, McGehee MD. Nanowire Solar Cells. Annual Review of Materials Research. 2011;41:269-95.

[6] Peng K, Xu Y, Wu Y, Yan Y, Lee S-T, Zhu J. Aligned Single-Crystalline Si Nanowire Arrays for Photovoltaic Applications. Small. 2005;1:1062-7.

[7] Lee S-H, Han S-H, Jung H-S, Shin H-S, Lee J, Noh JH, et al. Al-Doped ZnO Thin Film: A New Transparent Conducting Layer for ZnO Nanowire-Based Dye-Sensitized Solar Cells. Journal of Physical Chemistry C. 2010;114:7185-9.

[8] Leem JW, Yu JS. Structural, optical, and electrical properties of AZO films by tilted angle sputtering method. Thin Solid Films. 2010;518:6285-8.

[9] Zang Z, Nakamura A, Temmyo J. Single cuprous oxide films synthesized by radical oxidation at low temperature for PV application. Opt Express. 2013;21:11448-56.

[10] Wenas WW, Riyadi S. Carrier transport in high-efficiency ZnO/SiO2/Si solar cells. Solar Energy Materials and Solar Cells. 2006;90:3261-7.

[11] Peng K-Q, Lee S-T. Silicon Nanowires for Photovoltaic Solar Energy Conversion. Advanced Materials. 2011;23:198-215.

[12] Kelzenberg MD, Boettcher SW, Petykiewicz JA, Turner-Evans DB, Putnam MC, Warren EL, et al. Enhanced absorption and carrier collection in Si wire arrays for photovoltaic applications. Nat Mater. 2010;9:239-44.

[13] Huang Z, Shimizu T, Senz S, Zhang Z, Zhang X, Lee W, et al. Ordered Arrays of Vertically Aligned [110] Silicon Nanowires by Suppressing the Crystallographically Preferred $<100>$ Etching Directions. Nano Letter. 2009;9:2519-25.

[14] Huang Z, Geyer N, Werner P, de Boor J, Gösele U. Metal-Assisted Chemical Etching of Silicon: A Review. Advanced Materials. 2011;23:285-308.

[15] Kim J, Han H, Kim YH, Choi S-H, Kim J-C, Lee W. Au/Ag Bilayered Metal Mesh as a Si Etching Catalyst for Controlled Fabrication of Si Nanowires. ACS Nano. 2011;5:3222-9.

[16] Battaglia C, Hsu C-M, Söderström K, Escarré J, Haug F-J, Charrière M, et al. Light Trapping in Solar Cells: Can Periodic Beat Random? ACS Nano. 2012;6:2790-7.

[17] Neamen DA. Semiconductor Physics and Devices: Mc Graw Hill; 2012.

[18] Jung Y, Vacic A, Perea DE, Picraux ST, Reed MA. Minority Carrier Lifetimes and Surface Effects in VLS-

Grown Axial p-n Junction Silicon Nanowires. Advanced Materials. 2011;23:4306-11.

[19] Kato Y, Adachi S. Synthesis of Si Nanowire Arrays in AgO/HF Solution and Their Optical and Wettability Properties. Journal of The Electrochemical Society. 2011;158:K157.

[20] Schmidt V, Wittemann JV, Senz S, Gösele U. Silicon Nanowires: A Review on Aspects of their Growth and their Electrical Properties. Advanced Materials. 2009;21:2681-702.

[21] Yuan G, Aruda K, Zhou S, Levine A, Xie J, Wang D. Understanding the Origin of the Low Performance of Chemically Grown Silicon Nanowires for Solar Energy Conversion. Angewandte Chemie International Edition. 2011;50:2334-8.

[22] Adachi MM, Anantram MP, Karim KS. Core-shell silicon nanowire solar cells. Sci Rep. 2013;3.

[23] Allen JE, Hemesath ER, Perea DE, Lensch-Falk JL, LiZ.Y, Yin F, et al. High-resolution detection of Au catalyst atoms in Si nanowires. Nat Nano. 2008;3:168-73.

[24] Mariani G, Scofield AC, Hung C-H, Huffaker DL. GaAs nanopillar-array solar cells employing in situ surface passivation. Nat Commun. 2013;4:1497. 


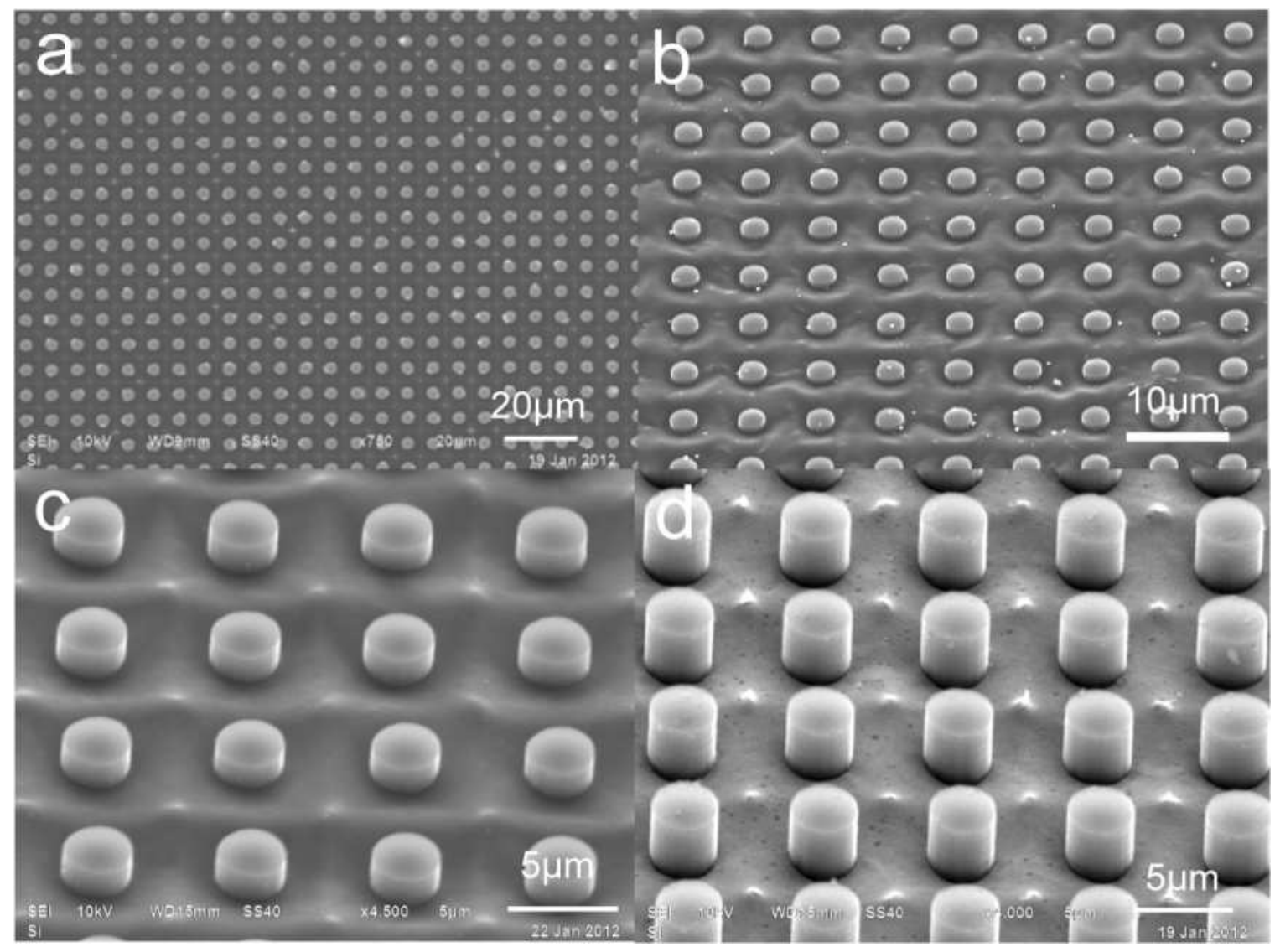

Fig. 1 SEM images of periodic Si pillar arrays synthesized by metal-assisted chemical etching method. (a) Top view of a large area Si pillar array. (b-d) $45^{\circ}$ tilted view of Si pillar arrays. The height of $\mathrm{Si}$ pillars is $0.68,1.15$, and $2.8 \mu \mathrm{m}$, respectively after etching for 7,15 and $30 \mathrm{~min}$. 

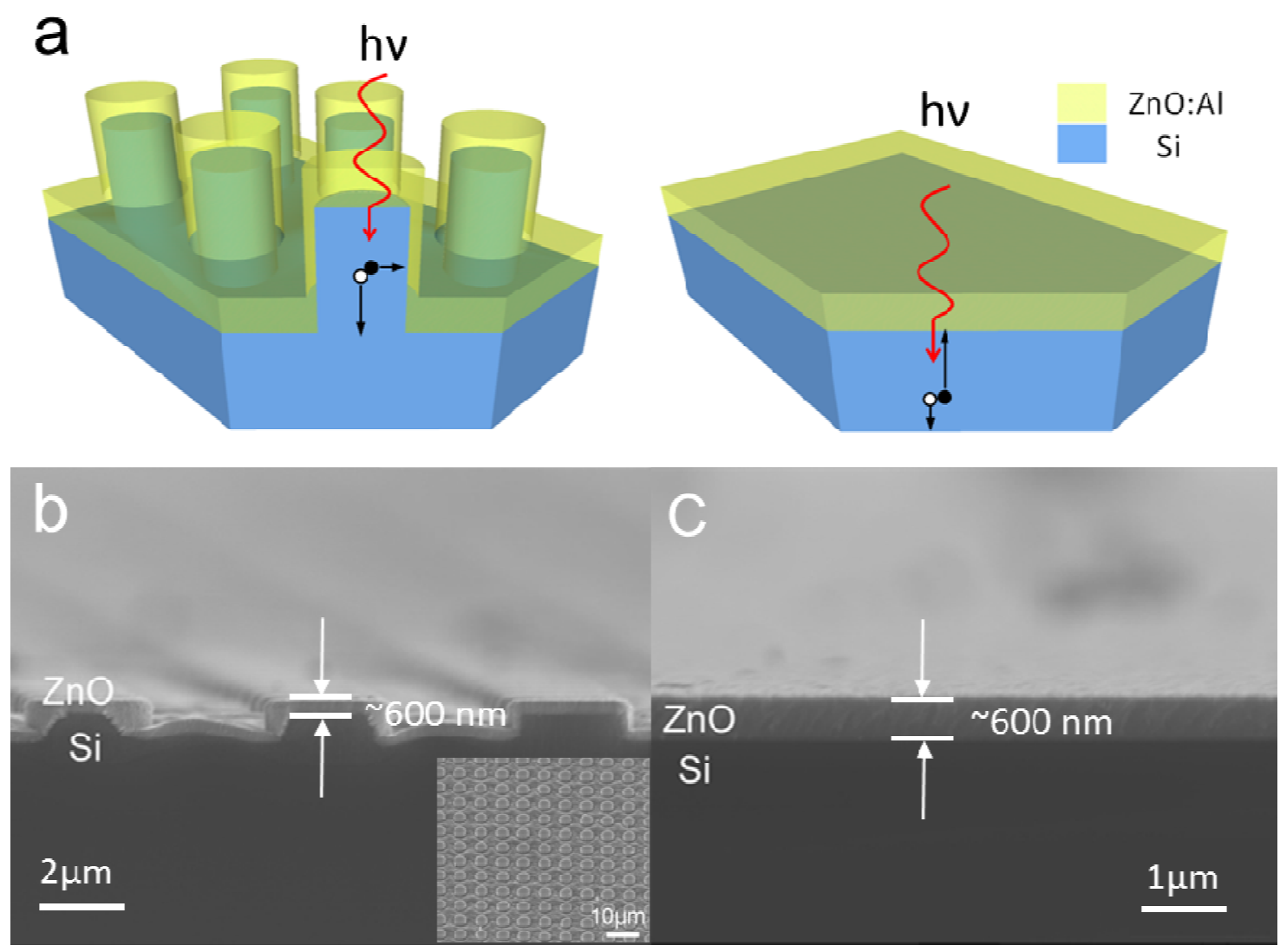

Fig. 2 (a) Schematic illustration of orthogonalizing light direction and charge separation direction in core-shell structure (left) as compared to the traditional planar structure (right). Cross-section SEM images of (b) core-shell structure and (c) planar structure $p-\mathrm{Si} / n-\mathrm{ZnO}$ heterojunction. Inset of (b) is $45^{\circ}$ tilted view of the core-shell structured $p$-Si $/ n-\mathrm{ZnO}$, showing a smooth and uniform $\mathrm{ZnO}$ coating. 


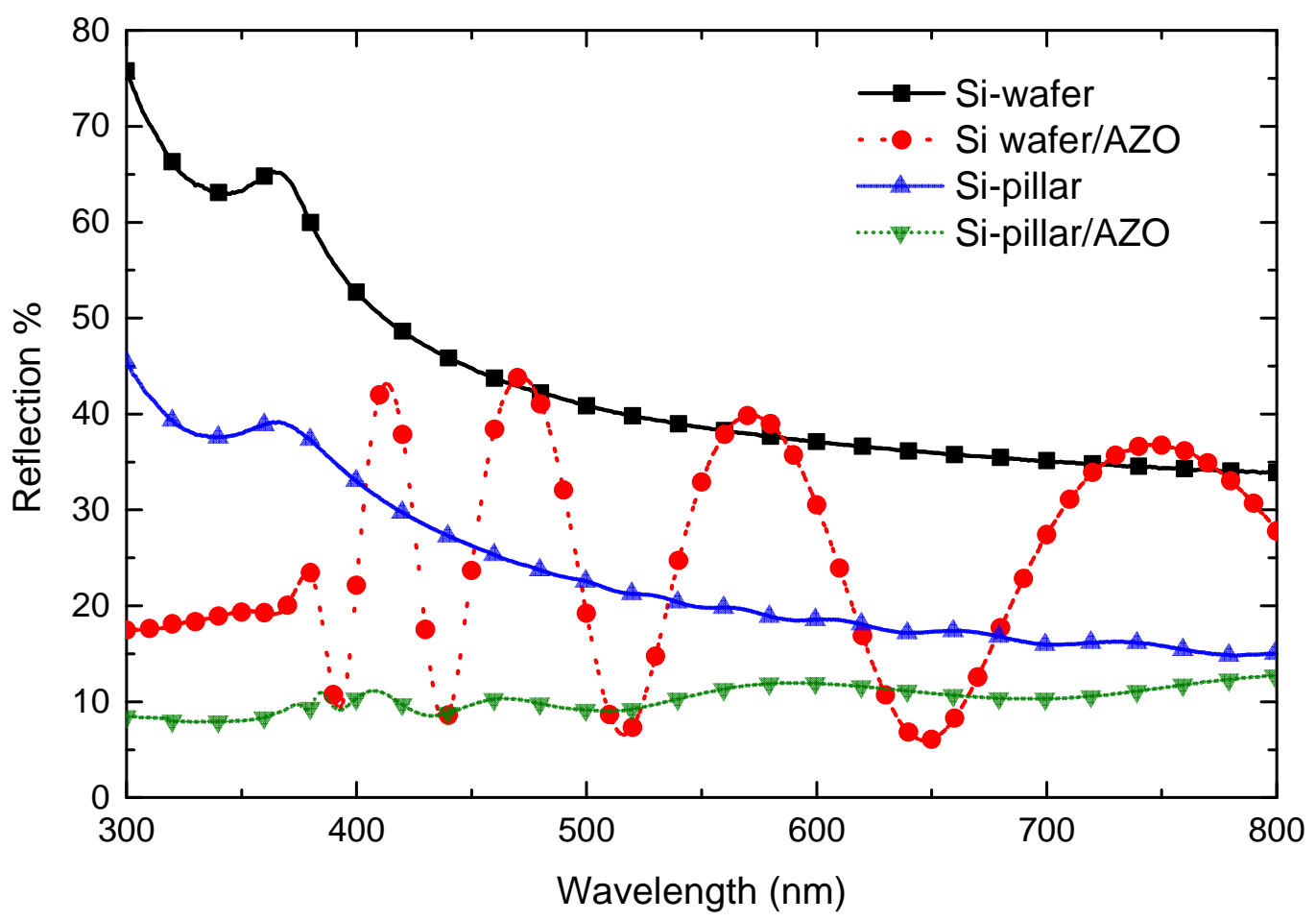

Fig. $3 \mathrm{UV}$-Vis reflection spectra of $\mathrm{Si}$ wafer, periodic Si pillar array, planar structured $\mathrm{Si} / \mathrm{ZnO}$ heterojunction and core-shell structured $\mathrm{Si} / \mathrm{ZnO}$ heterojunction. The core-shell structure shows the lowest reflectance in a wide wavelength range. 

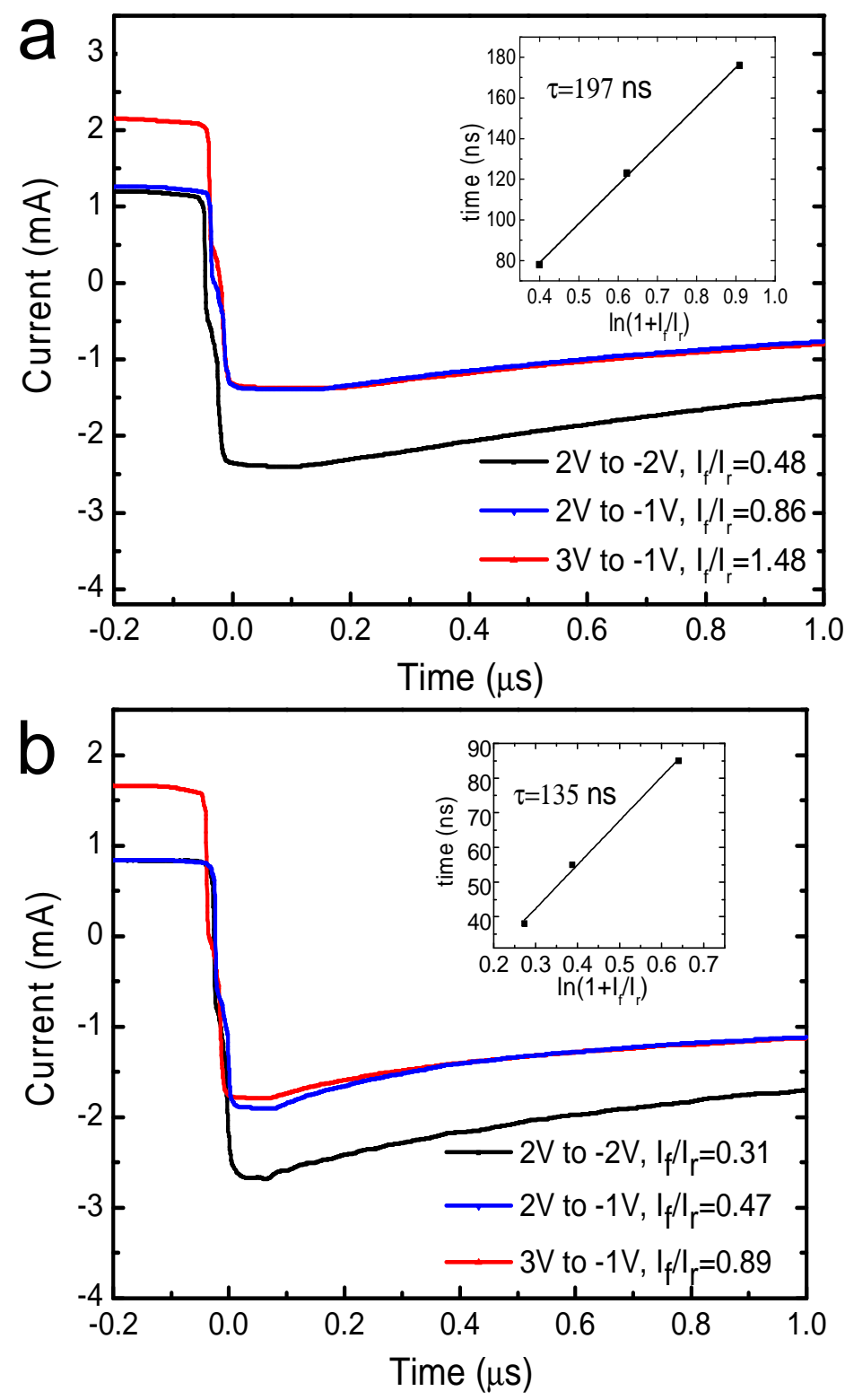

Fig. 4 (a) RRT characterizations of planar structure $\mathrm{Si} / \mathrm{ZnO}$ heterojunction. Inset: Storage time $t_{s}$ versus $\ln \left(1+I_{f} / I_{r}\right)$, the slope gives the minority carrier life time $\sim 197 \mathrm{~ns}$. (b) RRT characterizations of core-shell structure $\mathrm{Si} / \mathrm{ZnO}$ heterojunction. Inset: Storage time $t_{s}$ versus $\ln \left(1+I_{f} / I_{r}\right)$. The minority carrier life time is $\sim 135 \mathrm{~ns}$. 

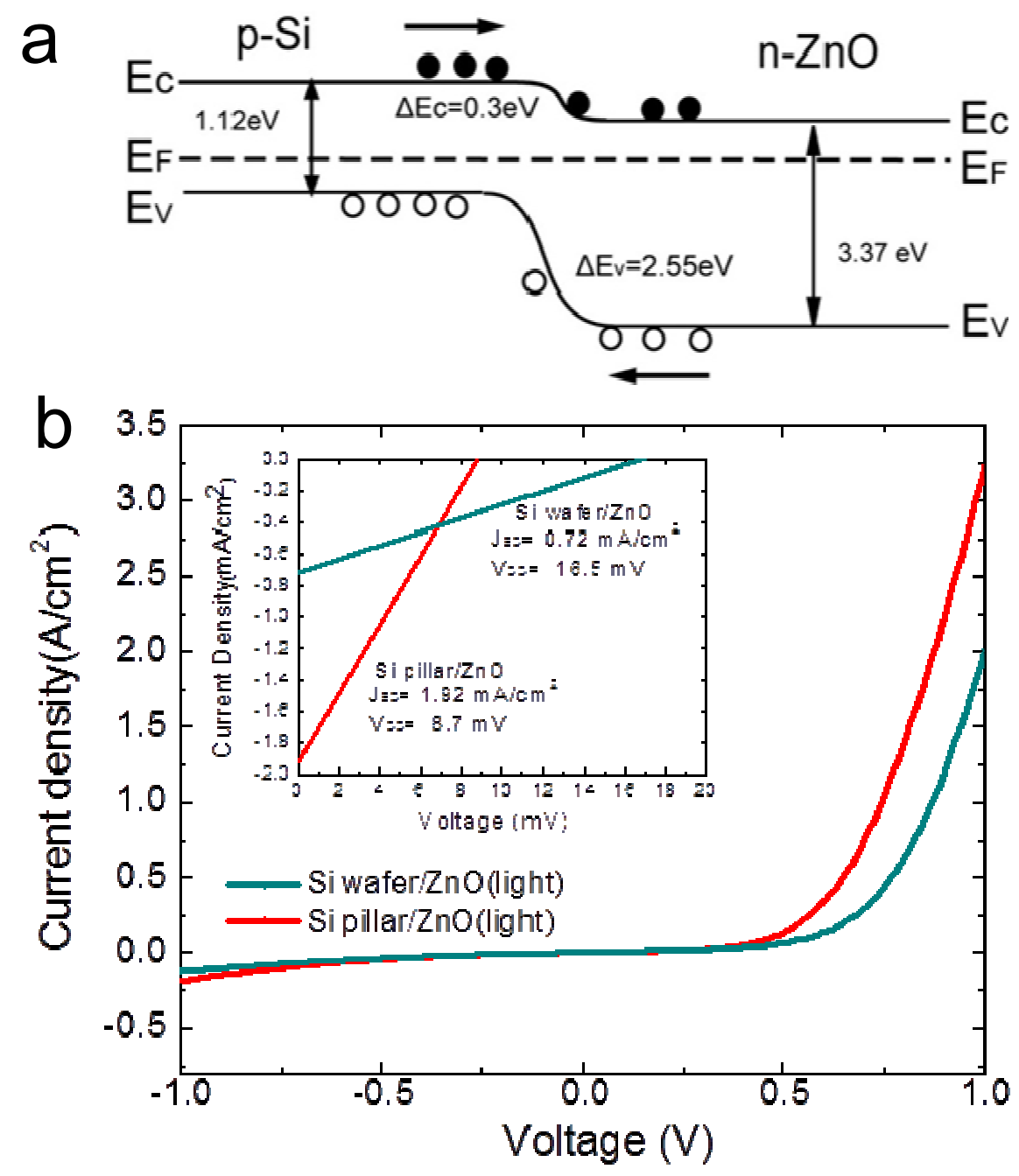

Fig. 5 (a) Energy band diagram of a $p-\mathrm{Si} / n-\mathrm{ZnO}$ heterojunction under solar light illumination. (b) J-V curve of planar structured and core-shell structured $p-\mathrm{Si} / n-\mathrm{ZnO}$ solar cells under light illumination. The inset shows enlarged view of current density versus voltage for both planar and core-shell structured solar cell under light illumination. $J_{S C}=0.72 \mathrm{~mA} / \mathrm{cm}^{2}, V_{O C}=16 \mathrm{mV}$ for planar structured solar cell and $J_{S C}=1.92 \mathrm{~mA} / \mathrm{cm}^{2}, V_{O C}=9 \mathrm{mV}$ for core-shell structured solar cell. 